\title{
Rare brain biopsy findings in a first ADEM-like event of pediatric MS: histopathologic, neuroradiologic and clinical features
}

\author{
Franziska Hoche $\cdot$ Sabine Pfeifenbring $\cdot$ Stefan Vlaho $\cdot$ \\ Mayyada Qirshi • Marius Theis • Wilfried Schneider • \\ Luciana Porto $\cdot$ Klaus Müller $\cdot$ Matthias Kieslich
}

Received: 9 January 2011 / Accepted: 15 February 2011/Published online: 10 March 2011

(c) Springer-Verlag 2011

\begin{abstract}
Pediatric MS tends to present more often with an acute onset and a polysymptomatic form of the disease, possibly with encephalopathy and large tumefactive lesions similar to those observed in some cases of acute disseminated encephalomyelitis (ADEM), which makes it more difficult to differentiate between an explosive and severe onset of MS vs. ADEM. An ADEM-like first demyelinating event can be the first attack of pediatric MS, but international consensus definitions require two or more non-ADEM demyelinating events for diagnosis of MS. In our patient KIDMUS MRI criteria for MS (Mikaeloff et al. J Pediatr 144:246-252, 2004a; Mikaeloff et al. Brain 127:1942-1947, 2004b) were negative at first attack, but Barkhof criteria for lesion dissemination in space in adults (Barkhof et al. 120:2059-2069, 1997), Callen modified MS-criteria and Callen MS-ADEM criteria for children (Callen et al. Neurology 72:961-967, 2009a; Callen et al.
\end{abstract}

F. Hoche $(\bowtie) \cdot$ S. Vlaho $\cdot$ M. Qirshi $\cdot$ M. Theis $\cdot$ M. Kieslich Department of Neuropediatrics, Goethe University Frankfurt, Theodor-Stern-Kai 7, 60590 Frankfurt, Germany

e-mail: franziska.hoche@kgu.de

F. Hoche $\cdot$ S. Pfeifenbring

Department of Neuropathology, University Medical Center

Göttingen, Robert-Koch Str. 40, 37099 Göttingen, Germany

\section{W. Schneider}

Department of Pediatric Intensive Care Medicine,

Goethe University Frankfurt, Frankfurt, Germany

L. Porto

Department of Neuroradiology, Goethe University Frankfurt, Frankfurt, Germany

K. Müller

Department of Neurology, Edinger Institute, Goethe University Frankfurt, Frankfurt, Germany
Neurology 72:968-973, 2009b) were positive suggesting pediatric MS. As the clinical course was devastating with non-responsiveness upon high-dose immune modulatory therapy and due to the absence of an alternative diagnosis other than demyelinating disease brain biopsy was performed. Brain biopsy studies or autopsy case reports of fulminant pediatric MS patients are extremely rare. Histopathology revealed an inflammatory demyelinating CNS process with confluent demyelination, indicating the likelihood of a relapsing disease course compatible with an acute to subacute demyelinating inflammatory disease. This pattern was corresponding to the early active multiple sclerosis subtype I of Lucchinetti et al. (Ann Neurol 47(6):707-717, 2000).

Keywords Pediatric multiple sclerosis - ADEM - MRI diagnostic criteria $\cdot$ Plasma exchange $\cdot$ International MS Study Group · MS histopathology

\section{Case report}

\section{Clinical course}

A 16-year old female presented with a 2-week history of numbness of the right facial skin, parasthesia of the right upper and lower lip, diplopia, parasthesia and palsy of the right hand, and incipient hemiparesis of the right leg. Upon admission the patient was afebrile and her medical history did not include an acute or prior infection and no past neurological illnesses. Her birth history and postnatal development had been unremarkable. Vital signs were stable and her mental status was intact. Physical examination revealed a lack of sensitivity of the right facial skin, pronounced diplopia when looking to the left, a slight 
weakness of the right upper extremity, a pronounced weakness of the right lower extremity, and weak dorsiflexion of the right foot.

Initial laboratory studies showed no abnormalities. Lumbar puncture and cerebrospinal fluid (CSF) analysis indicated a normal opening pressure with macroscopically clear CSF, total protein levels of $369 \mathrm{mg} / \mathrm{l}$ with an albumin level of $253 \mathrm{mg} / \mathrm{l}$, lactate of $1.38 \mathrm{mmol} / \mathrm{l}$, and glucose of $58.7 \mathrm{mg} / \mathrm{dl}$. White blood cell count was elevated at $26 / \mu \mathrm{l}$ including a majority of immunoreactive lymphocytes and monocytes. No erythrocytes were present. The sample showed type-3-oligoclonal bands accompanied by intrathecal synthesis of immunoglobulin G. The CSF/Serum glucose index was normal. Gram stains, blood cultures, and serological analysis were negative. The pleocytosis in the CSF led to the administration of an antibiotic and antiviral medication from day one. Cranial magnetic-resonanceimaging (MRI) revealed extensive tissue defects with restricted diffusion indicating an acute demyelinating process. Thereupon treatment with high-dose methylprednisolone ( $1 \mathrm{~g} /$ day $)$ was started.

On the second day after hospital admission the patient's neurological status declined progressively. In the morning she displayed anxiety, states of sudden arousal, as well as reduced vigilance and consciousness. The hemiparesis of the right lower leg became evident and she was unable to ambulate. Reflexes as well as superficial, noxious and deep sensation of the lower limbs were absent. Visual acuity was limited to hand movement and finger counts with bitemporal hemianopia. She showed dysphagia and dysarthria, as well as incipient urinary and stool incontinence. Visual evoked potential amplitudes were found to be reduced and latencies of bilateral contracortical waves were clearly prolonged. At the end of this day, the patient was blind, presenting spastic tetraparesis, complete urinary and stool incontinence, and complete loss of consciousness. Sudden respiratory paralysis led to admission to the intensive care unit where tracheal intubation and assisted ventilation were initiated. The patient's condition declined rapidly. The need for brain biopsy became inevitable due to the lack of a differential diagnosis other than demyelinating disease, as well as due to the patient's non-responsiveness towards high-dose corticosteroid treatment and plasmapheresis. Following this, therapy with double the dosage of methylprednisolone ( $2 \mathrm{~g} / \mathrm{day})$ was recommended for five more days.

\section{Neuroimaging findings}

On day one of hospital admission an initial MRI scan of the brain was performed. MR images were acquired on a 3 tesla magnet with slice thickness between 2 and $5 \mathrm{~mm}$ and an interslice gap of up to $2 \mathrm{~mm}$. T2-weighted images showed multiple periventricular and deep white matter focal lesions (Fig. 1a), one juxtacortical lesion of the left precentral region, involvement of the corpus callosum as well as one hyperintense lesion within the right brachium pontis (Fig. 1b) associated diffuse hyperintense changes. Fluid attenuated inversion recovery (FLAIR) sequence revealed multiple hyperintense areas, including oval lesions within the periventricular white matter (PVWM) and the corpus callosum (Fig. 1c). The lesions were oriented perpendicular to the long axis of the ventricular system. Diffusion weighted image (DWI) showed restricted diffusion as a feature of inflammatory disease activity. Large lesions were seen in the centrum semiovale bilaterally with ill-defined borders, presence of mass defect and marked restriction in diffusion images (Fig. 1d). Apparent diffusion coefficient (ADC) maps (Fig. 1e) clearly showed reduced signal, indicating the presence of cytotoxic edema (Axer et al. 2005).

T1-weighted images after contrast (gadolinium) showed enhancement within the PVWM (Fig. 1f) as well as in the right cerebellar peduncle corresponding to the described lesions of T2 and FLAIR sequences representing areas of active demyelination. All hyperintense lesions in T2 weighted imaging described above were concordant with hypointense areas in $\mathrm{T} 1$ weighted images compared with white matter and resembling gray matter. $\mathrm{T} 2 *$ sequences showed no sign of intracranial bleeding or vascular lesions. There was no evidence for intracranial neoplasm or vasculitis. Cerebral fluid interspaces were of normal agematched width and there was no sign of elevated cranial pressure.

Follow-up MRI performed on day four of hospital admission showed an increase of the tumefactive demyelinating lesions, particularly in the PVWM. The number of diffuse ovoid lesions in T2 and FLAIR images increased with further spread towards the truncus and splenium of the corpus callosum. There was a progression of the active demyelinating lesions with mass defect including the involvement of the subcortical white matter. Axial T1weighted image showed large bilaterally hypointense lesions with signs of cystic necrotic changes concordant with hyperintense areas in axial $\mathrm{T} 2 \mathrm{w}$ sequences (Fig. 1g). The known lesion of the right cerebellar peduncle was unchanged.

Altogether, the patient was positive when applying the following diagnostic MRI criteria: Barkhof criteria for lesion dissemination in space in adults (Barkhof et al. 1997), Callen modified MS-criteria for children (Callen et al. 2009a, b), and Callen MS-acute disseminated encephalomyelitis (MS-ADEM) criteria for children (Callen et al. 2009a, b). Negative results were obtained when applying KIDMUS criteria to our patient (Mikaeloff et al. 2004a, b). 

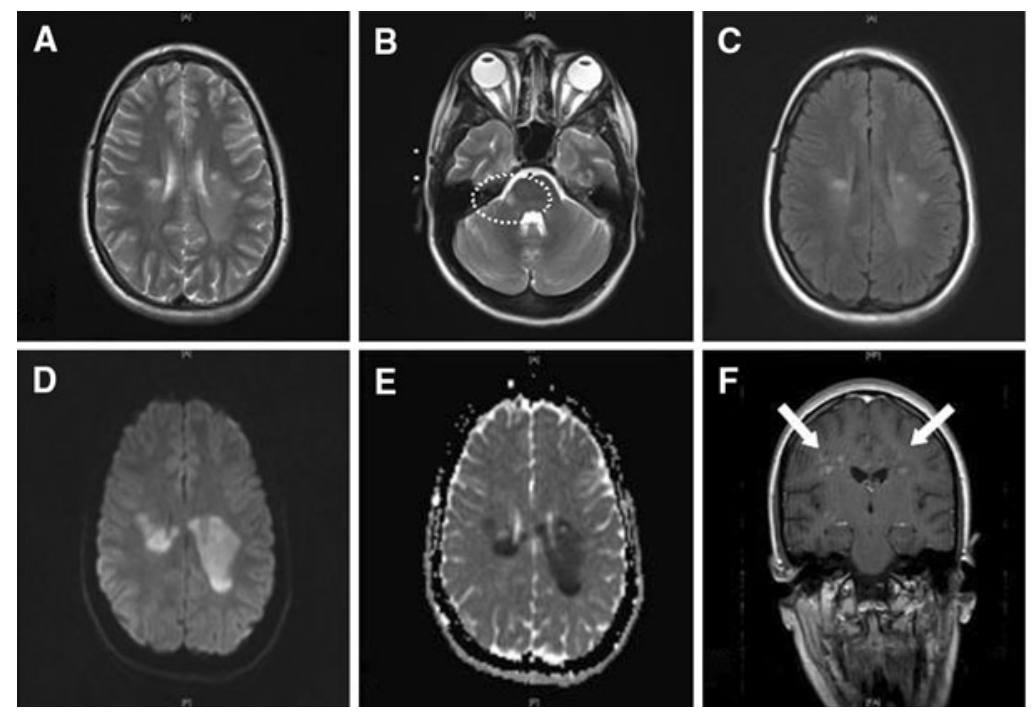

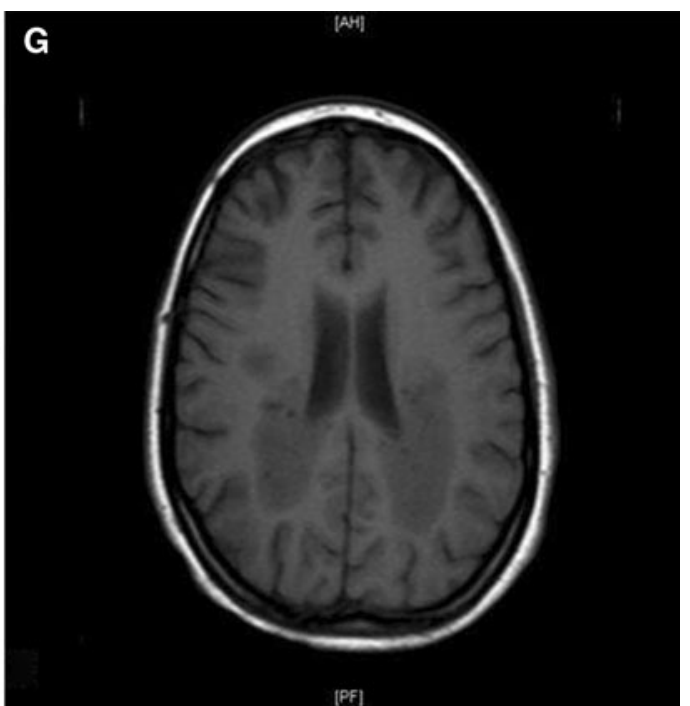

Fig. 1 a Axial T2-weighted image of the initial disease stage shows multiple periventricular and deep white focal lesions associated with diffuse hyperintense changes. b T2-weighted image showing hyperintensity of the pons. $\mathbf{c}$ The focal lesions were hyperintense on FLAIR image. d Diffusion-weighted image showed restricted diffusion, confirmed by the Apparent diffusion coefficient (ADC) maps. e ADC maps showing reduced signal, indicating the presence of cytotoxic edema. f T1-weighted image of gadolinium enhancing lesions within the centrum semiovale of both hemispheres. g Axial T1-weighted image showing large bilaterally hypointense lesion with small areas of tissue defect concordant with hyperintensity in T2-weighted image

\section{Neuropathological findings}

Materials and methods

Neuropathological examination: Intraoperative diagnostics consisted of cytological evaluation of smears stained with methylene blue. Paraffin-embedded tissue was investigated using classical hemalaun eosin (HE) stains followed by periodic acid Schiff stainings, Klüver-Barrera and reticulin stainings as well as immunohistochemistry for CD68 (microglia/macrophages), CD4 (T-helper cells), CD8 (cytotoxic T-cells), CD20 (B-cells), neurofilament (axonal structures), glial fibrillary acid protein (reactive astrocytes), the monoclonal antibody MIB-1 (proliferation index), C9neo (complement deposits), and CNPase (2',3'-cyclic nucleotide-3-phosphohydrolase). Evaluation of the histological and immunohistochemical stainings and photographic documentation were performed using an Olympus BX-40 light microscope (Hamburg, Germany). Pictures were taken with a digital camera (Olympus, DP72, Hamburg, Germany). Furthermore electron microscopy was performed of selected areas using a FEI Tecnai G2 Spirit Biotwin electron microscope (Hillsboro, OR).

\section{Results}

Stereotactic biopsy tissue showed signs of brain edema accompanied by astrogliosis and a moderate increase in cellularity (Fig. 2a). Single Creutzfeldt-Peters cells were observed (Fig. 2a; inset). Besides, small necrotic foci were present. Myelin-specific stainings revealed dystrophic axonal spheroids and severe loss of myelin (Fig. 2b). Immunohistochemical examination confirmed the demyelinating character of the lesion in which a massive infiltration of foamy macrophages and activated microglial cells was seen (Fig. 2c) while axons were mainly preserved (Fig. 2d). The main population of diffusely intermingled inflammatory cells consisted of CD8-positive cytotoxic T-cells. No hints of a vasculitis or other inflammatory CNS diseases as discussed in the clinical differential diagnoses were seen. No complement deposits could be detected in the biopsies. On an ultrastructural level, both active demyelination (so-called myelin stripping by monocytic cells; Fig. 2e) and completely demyelinated axons (socalled nude axons; Fig. 2f) were observed. Especially, the prominent signs of myelin stripping indicate an early active demyelinating lesion. There were no classical morphological findings supporting the differential diagnosis of an acute demyelinating encephalomyelitis disseminata such as a perivenular demyelination pattern (Pittock et al. 2005). In contrast, an MS typical confluent demyelination pattern was observed. Thus the T-cell/macrophage-mediated myelin destruction without deposition of activated complement on degenerating myelin sheaths or within the cytoplasm of infiltrating macrophages could be classified as pattern I according to Lucchinetti et al. (Lucchinetti et al. 2000). 
Fig. 2 Histology/

immunohistochemistry/electron microscopy. a The biopsy specimen showed CNS tissue with a moderate increase in cellularity, reactive gliosis, edema, and some dilated blood vessels (HE); single

Creutzfeldt-Peters cells were seen (inset). b A severe loss of myelination was seen with only few remaining myelinated axons (Klüver-Barrera). c Massive macrophage/ microglia infiltration was observed in the stereotactic biopsies (CD68) while $\mathbf{d}$ axonal structures were preserved (neurofilament). At the ultrastructural level, both e active demyelination (arrow indicating a macrophage surrounding an axon) and almost $\mathbf{f}$ completely demyelinated axons (arrow) were present
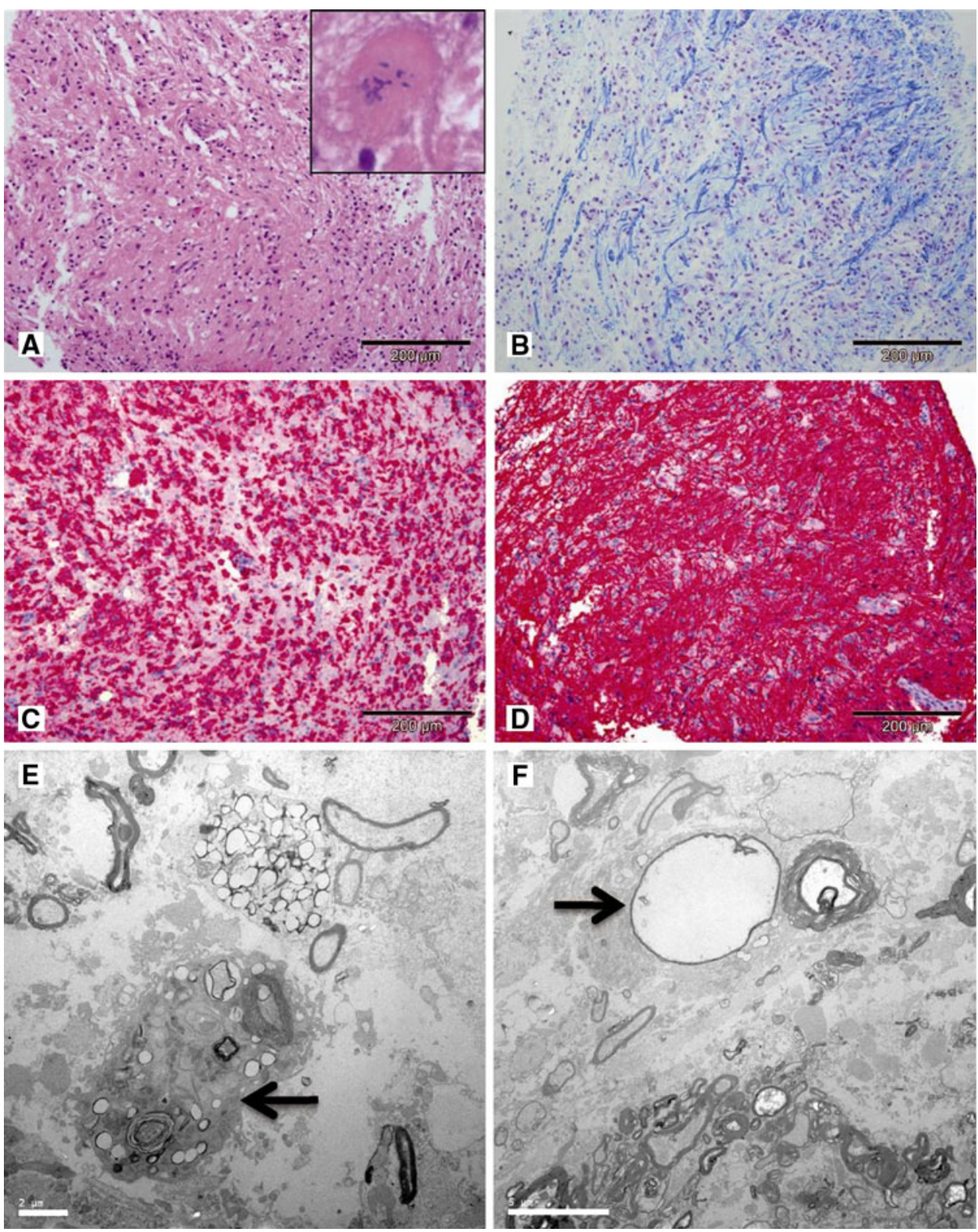

\section{Clinical outcome}

The patient was extubated on day 19 after hospital admission. On day 29 of hospital admission she was transferred to a neurorehabilitative hospital. The MRI control 8 weeks after therapeutic treatment with plasmapheresis for 7 days and administration of double the dosage of methylprednisolone for 5 days showed complete regression of the enhancing lesions with no restricted diffusion on DWI.

Six months after the first event, the patient showed a second monosymptomatic event without encephalopathy suffering from numbness of the left lower leg. No MRI was performed then. Another 6 months later the patient presented with a third monosymptomatic demyelinating attack without signs of encephalopathy involving new areas of the CNS. She displayed sensory disturbances in the roof of the mouth, as well as of the right side of the face. The MRI showed one new gadolinium-enhancing lesion located within the right pons near the rootlets of the trigeminal nerve (not shown) fulfilling McDonald revised MS diagnostic criteria of lesion dissemination in time (Polman et al. 2005). T1 weighted images showed persisting hypointense lesions, so called persistent black holes (Sahraian et al. 2010) of the PVWM.

The patient received methylprednisolone ( $1 \mathrm{~g} /$ day) for 3 days whereupon symptoms decreased. 


\section{Discussion}

Pediatric MS tends to present more often with an acute onset and a polysymptomatic form of the disease, possibly with encephalopathy and large tumefactive lesions similar to those observed in some cases of ADEM, which makes it more difficult to differentiate between an explosive and severe onset of MS vs. ADEM (Mikaeloff et al. 2004a, b, 2007; Dale and Pillai 2007). An ADEM-like first demyelinating event can be the first attack of pediatric MS, but international consensus definitions require two or more non-ADEM demyelinating events for diagnosis of MS (Krupp et al. 2007). MRI is a useful tool in making the diagnosis of acute inflammatory demyelination and might be helpful in recognizing patients who are at risk of future attacks of demyelination. When polyfocal demyelination is accompanied by encephalopathy, the clinical diagnosis of ADEM is applied (Krupp et al. 2007). MRI features of ADEM typically include widespread, bilateral, and asymmetric involvement of supratentorial and infratentorial white matter and deep gray nuclei as partially seen in our patient. On the other hand, in approximately $20 \%$ of children who are initially diagnosed with ADEM subsequent multiple attacks lead to the diagnosis of MS (Mikaeloff et al. 2004a, b, 2007; Dale and Pillai 2007; Neuteboom et al. 2008; Banwell et al. 2007).

Two sets of MRI criteria designed for predicting conversion to MS after a first demyelinating event, the Barkhof MRI-criteria for adults (Barkhof et al. 1997) and the KIDMUS criteria for children (Mikaeloff et al. 2004a, b), were applied to our patient at first demyelinating event (Table 1). While Barkhof criteria of lesion dissemination in space turned positive during the patient's first demyelinating event, KIDMUS criteria remained negative due to the required presence of a solely discrete lesion pattern (Mikaeloff et al. 2004a, b). Of interest here is that in a recent study by Callen et al. (2009a, b) $65 \%$ of pediatric patients with first onset of MS had at least one large lesion ( $\geq 2 \mathrm{~cm}$ in diameter) (Callen et al. 2009a, b). The occurrence of large lesions in children with MS has been reported previously (Gallucci et al. 2001; Ruggieri et al. 2004). In another comparative study both sets of criteria, Barkhof and KIDMUS criteria, have been shown to have a high specificity and positive predictive value in predicting conversion to a clinically definite MS but show low sensitivity in children under age of 10 years (Ketelslegers et al. 2010).

Hahn et al. (2004) already reported that adult-based MRI diagnostic criteria were not as sensitive to the appearance of MS in children. This led Callen et al. (2009a, b) to a revision and modification of the McDonald criteria for lesion dissemination in space in order to better distinguish pediatric MS from nondemyelinating diseases in childhood. The criteria by Callen differed from the McDonald criteria for MS in adults by requiring two or more of (rather than three criteria) (1) $\geq \mathrm{T} 2$ rather than more than $9 \mathrm{~T} 2$ lesions (2) $\geq 2$ periventricular lesions rather than $\geq 3$, and ( 3$) \geq 1$ brainstem rather than $\geq 1$ infratentorial lesion. The criterion of $\geq 1$ juxtacortical lesion was not found to contribute to the pediatric MRI criteria, and gadolinium-enhancing lesions were not included because many children of the cohort studied by Callen et al. did not receive gadolinium. Application of adult MS McDonald criteria for dissemination in space to the pediatric cohort of Callen et al. produced a sensitivity of only $76 \%$ as compared with the $85 \%$ sensitivity of the proposed criteria. Applying the Callen modified MS- criteria to our patient we also received a positive result suggesting the diagnosis of MS (Table 1). Though Callen noted that these new pediatric MS criteria were met not only by $75 \%$ of the 28 children with first attack of MS examined by this study but at the same time also by $75 \%$ of the children with ADEM indicating that the criteria were a valid means of distinguishing MS from nondemyelinating acute neurologic disorders in children but that they might lack the specificity to distinguish between pediatric MS and ADEM. These results led him to a second revision of the diagnostic MRI criteria available in order to determine MRI diagnostic criteria that better differentiated between pediatric MS and monophasic demyelination (ADEM) (Callen et al. 2009a, b). Out of a comprehensive quantitative and qualitative analysis of MRI obtained at a first attack of pediatric MS and ADEM, Callen et al. (2009a, b) proposed the following criteria: any two of (1) absence of a diffuse bilateral lesion pattern, (2) presence of black holes, and (3) presence of two or more periventricular lesions. Applying these criteria MS patients at first attack could be distinguished from monophasic ADEM patients with an $81 \%$ sensitivity and a $95 \%$ specificity. Using these criteria in our patient they also were positive indicating a relapsing demyelinating disease course of MS.

Nevertheless, in our patient the clinical course was devastating, resulting in stereotactic brain biopsy. Histopathology revealed an inflammatory demyelinating CNS process with a confluent demyelination, inflammation, reactive gliosis, and relative axonal preservation. A massive infiltration by foamy macrophages and a few T-cells could be observed. LFB- and CNPase-positive myelin degradation products were detectable within the cytoplasm of some macrophages, indicating an early active demyelinating lesion. Small areas of remyelination could be seen. Deposition of activated complement was absent. No hints for a vasculitis or other inflammatory CNS diseases as discussed in the clinical differential diagnoses were seen, and there were no classical morphological findings supporting the differential diagnosis of an acute demyelinating 
Table 1 Comparison of different MS-MRI diagnostic criteria applied to our patient at first demyelinating event

\begin{tabular}{ll}
\hline Different MRI-criteria for MS published recently & $\begin{array}{l}\text { Patient's first } \\
\text { demyelinating attack }\end{array}$ \\
\hline KIDMUS (Mikaeloff et al. 2004a, b) & \\
All of: & \\
$\geq 1$ lesion perpendicular to long axis of corpus callosum & Yes \\
Presence of solely discrete lesions ( $\leq 2 \mathrm{~cm}$ in diameter) & No \\
McDonald (Barkhof et al. 1997) & \\
At least three of: & \\
$\geq 9$ T2 lesions or $\geq 1$ gadolinium enhancing lesion & Yes \\
$\geq 3$ periventricular & Yes \\
$\geq 1$ juxtacortical & Yes \\
$\geq 1$ infratentorial (or spinal) lesion & Yes \\
Callen modified MS-criteria (Callen et al. 2009a, b) & \\
At least two of: & \\
$\geq 5$ T2 lesions & Yes \\
$\geq 2$ periventricular & Yes \\
$\geq 1$ brainstem & Yes \\
Callen MS-ADEM criteria (Callen et al. 2009a, b) & \\
Any two of: & \\
Absence of diffuse bilateral lesion pattern & No \\
Presence of black holes & Yes \\
Presence of two or more periventricular lesions & \\
\hline
\end{tabular}

encephalomyelitis disseminata such as a perivenular demyelination pattern (Pittock et al. 2005). The present case was compatible with an acute to subacute demyelinating inflammatory disease corresponding to the early active multiple sclerosis subtype I according to the classification of Lucchinetti et al. (2000). The presence of CNP within macrophages indicated an early demyelination. Subtype II (antibody/complement associated) could be ruled out by the lack of C9neo deposits. Since there were oligodendrocytes forming new thin myelin sheets within the lesions, subtypes III (oligodendrogliopathy) and IV (oligodendrocyte degeneration in the periplaque white matter) were as well unlikely.

Brain biopsy studies or autopsy case reports of pediatric MS patients are rare. Even more rare are biopsy specimen out of large ill defined ADEM like MS-lesions in a pediatric/adolescent patient. Up to now there are no reports or studies on specific histopathological findings in pediatric MS (Pohl 2008). Pathological findings in adult MS cohorts demonstrate heterogeneity, classifiable into the upper mentioned four subtypes. Though, it remains unclear whether these four distinct pattern are generally applicable to pediatric MS patients.

From clinical point of view according to the consensus definitions of the International Pediatric MS Study Group, our patient did not fulfill the diagnostic criteria for MS at first onset of the disease (Krupp et al. 2007) due to the absence of multiple demylinating events separated in time and space and due to the presence of encephalopathy at first attack. Consensus definitions require a third clinical and/or new T2-lesions at least 3 months from the second event to be diagnosed with MS. These criteria were met by the patient 12 months after initial attack. The patient did not meet recurrent or multiphasic ADEM criteria at second and third event (Krupp et al. 2007).

Taken together, neuroradiologic MRI criteria were inconsistent with positive Callen MS-ADEM criteria (Callen et al. $2009 \mathrm{a}, \mathrm{b})$ which have been shown to possibly be the most useful MRI criteria for differentiating pediatric first MS from ADEM (Ketelslegers et al. 2010); histopathologic findings indicated an early active multiple sclerosis subtype I (Lucchinetti et al. 2000), but clinical features contradicted the presence of MS and indicated ADEM (Krupp et al. 2007). Regarding the rarity of fulminant MS variants, there is no uniform treatment strategy. It is known that patients with MS may benefit from early aggressive therapy during the initial clinical event (Young et al. 2010). Hence, an intensified immunomodulatory treatment might have prevented a continuing inflammatory disease activity in our patient.

Our findings reflect the current problem of diverse and inconsistent findings in pediatric MS. The importance of further differentiating MRI-criteria for children under 10 years (or child vs. adolescent) has been noted by other researchers also (Callen et al. 2009a, b) and might be underlined by our findings. It is unclear whether lesion pattern of pediatric MS might change with age of onset, 
and possibly in our patient there already was a more adultspecific lesion pattern leading to a positive matching of pediatric and adult MRI criteria. Second, Callen et al. (2009a, b) proposed further work up on a suggested shift of diffuse lesion pattern of a first ADEM-like MS event from large mass-like lesions to more MS-like, single-shaped lesions in older children and adolescents and that further research on the presence of gadolinium-enhancing lesion pattern in children and adults with MS was required. Fourth, pediatric histopathologic and CSF biomarker studies might be necessary to further discuss differences in neurorehabilitative potential of pediatric MS patients, possible implications for treatment strategies, and concordance with current histopathological classification in adults (Lucchinetti et al. 2000).

Our report aims at broadening the understanding of the clinical course, histopathologic and neuroradiologic features of pediatric ADEM-like first demyelinating attacks converting to MS during disease course. In our patient we had the rare chance to investigate stereotactic brain biopsy specimen in a pediatric patient with such initial fulminant variant of MS. We find it important to share the experience of different treatment procedures in fulminant MS variants with other researchers and hope to contribute diagnostic data for future revisions on MS classifications in order to further specify MS patient cohorts.

Acknowledgments We are indebted to Mr Karsten Kessler and Miss Mirjam Laufs for carefully reading and correcting the manuscript. In addition, we thank the patient and her family for giving their consent to the publication of these clinical data.

Conflict of interest The authors declare that there are no conflicts of interest.

\section{References}

Axer H, Ragoschke-Schumm A, Böttcher J, Fitzek C, Witte OW, Isennmann S (2005) Initial DWI and ADC imaging may predict outcome in acute disseminated encephalomyelitis: report of two cases of brain stem encephalitis. J Neurol Neurosurg Psychiatry 76:996-998. doi:10.1136/jnnp.2004.045500

Banwell B, Ghezzi A, Bar-Or A, Mikaeloff Y, Tardieu M (2007) Multiple sclerosis in children: clinical diagnosis, therapeutic strategies, and future directions. Lancet Neurol 6:887-902. doi:10.1016/S1474-4422(07)70242-9

Barkhof F, Filippi M, Miller DH et al (1997) Comparison of MRI criteria at first presentation to predict conversion to clinically definite multiple sclerosis. Brain 120:2059-2069. doi:10.1093/ brain/120.11.2059

Callen DJ, Shroff MM, Branson HM et al (2009a) MRI in the diagnosis of pediatric multiple sclerosis. Neurology 72:961-967. doi:10.1212/01.wnl.0000338629.01627.54

Callen DJ, Shroff MM, Branson HM et al (2009b) Role of MRI in the differentiation of ADEM from MS in children. Neurology 72:968-973. doi:10.1212/01.wnl.0000338630.20412.45
Dale RC, Pillai SC (2007) Early relapse risk after a first CNS inflammatory demyelination episode: examining international consensus definitions. Dev Med Child Neurol 49:887-893. doi: 10.1111/j.1469-8749.2007.00887.x

Gallucci M, Caulo M, Cerone G, Masciocchi C (2001) Acquired inflammatory white matter disease. Childs Nerv Syst 17:202210. doi: $10.1007 / \mathrm{s} 003810000331$

Hahn CD, Shroff MM, Blaser S, Banwell BL (2004) MRI criteria for multiple sclerosis: evaluation in a pediatric cohort. Neurology 62:806-808. doi:10.1212/01.WNL.0000113723.36715.A6

Ketelslegers IA, Neutebom RF, Boon M, Catsman-Berrevoets CE, Hintzen RQ, The Dutch Pediatric MS Study Group (2010) A comparison of MRI criteria for diagnosing pediatric ADEM and MS. Neurology 3:1412-1415. doi:10.1212/WNL.0b013e3181dc138b

Krupp LB, Banwell B, Tenebaum S (2007) Consensus definitions proposed for pediatric multiple sclerosis and related disorders. Neurology 68:S7-S12. doi:10.1212/01.wn1.0000259422.44235.a8

Lucchinetti C, Brück W, Parisi J, Scheithauer B, Rodriguez M, Lassmann H (2000) Heterogeneity of multiple sclerosis lesions: implications for the pathogenesis of demyelination. Ann Neurol 47(6):707-717. doi:10.1002/1531-8249(200006)47:6<707:AIDANA3 $>3.0 . C O ; 2-Q$

Mikaeloff Y, Suissa S, Vallee L et al (2004a) First episode of acute CNS inflammatory demyelination in childhood: prognostic factors for multiple sclerosis and disability. J Pediatr 144:246252. doi:10.1016/j.jpeds.2003.10.056

Mikaeloff Y, Adamsbaum C, Husson B et al (2004b) MRI prognostic factors for relapse after acute CNS inflammatory demyelination in childhood. Brain 127:1942-1947. doi:10.1093/brain/awh218

Mikaeloff Y, Caridade G, Husson B, Suissa S, Tardieu M (2007) Acute disseminated encephalomyelitis cohort study: prognostic factors for relapse. Eur J Paediatr Neurol 11:90-95. doi: 10.1016/j.ejpn.2006.11.007

Neuteboom RF, Boon M, Catsman Berrevoets CE et al (2008) Prognostic factors after a first attack of inflammatory CNS demyelination in children. Neurology 71:967-973. doi:10.1212/ 01.wnl.0000316193.89691.e1

Pittock SJ, McClelland RL, Achenbach SJ, Konig F, Bitsch A, Brück W, Lassmann H, Parisi JE, Scheithauer BW, Rodriguez M, Weinshenker BG, Lucchinetti CF (2005) Clinical course, pathological correlations, and outcome of biopsy proved inflammatory demyelinating disease. J Neurol Neurosurg Psychiatry 76:1693-1697. doi:10.1136/jnnp.2004.060624

Pohl D (2008) Epidemiology, immunopathogenesis and management of pediatric central nervous system inflammatory demyelinating conditions. Curr Opin Neurol 21:366-372. doi:10.1097/WCO. 0b013e3282fd172b

Polman CH, Rheingold SC, Edan G, Filippi M, Hartung HP, Kappos L, Lublin FD, Metz LM, Mc Farland HF, O'Connor PW, Sandberg-Wollheim M, Thompson AJ, Weinshenker BG, Wolmsky JS (2005) Diagnostic criteria for multiple sclerosis: 2005 revisions to the "McDonald Criteria". Ann Neurol 58:840846. doi:10.1002/ana.20703

Ruggieri M, Iannetti P, Polizzi A, Pavone L, Grimaldi LM (2004) Multiple sclerosis in children under 10 years of age. Neurol Sci 25(suppl 4):S326-S335. doi:10.1007/s10072-004-0335-Z

Sahraian MA, Radue EW, Haller S, Kappos L (2010) Black holes in multiple sclerosis: definition, evolution, and clinical correlations. Acta Neurol Scand 122:1-8. doi:10.1111/j.1600-0404.2009. 01221.x

Young NP, Weinshenker BG, Parisi J, Scheithauer B, Giannini C, Roemer SF, Thomson KM, Mandrekar JN, Erickson BJ, Luchinetti C (2010) Perivenous demyelination: association with clinically defined acute disseminated encephalomyelitis and comparison with pathologically confirmed multiple sclerosis. Brain 133:333-348. doi:10.1093/brain/awp321 